Check for updates

Cite this: RSC Adv., 2018, 8, 22687

\title{
Development and evaluation of pymetrozine controlled-release formulation to control paddy planthopper
}

\author{
Wei-Ming Xu, (D)* Ming Zhang, Kun Wei, Yan Chen, † Qin Liu, Wei Xue, Lin-Hong Jin,
} Ming He, Zuo Chen and Song Zeng

Continuous outbreaks of rice planthoppers in rice-growing regions in China indicates the importance of redesigning several planthopper management programs. Chemical control remains the main strategy for planthopper control in China and other subtropical and temperate regions. Most common chemical insecticides are emulsifiable concentrates, suspension concentrates, soluble concentrates, and wettable powders. These insecticides are applied by dusting or spraying using simple equipment. The active ingredient, with short effectiveness time, is degraded rapidly in natural paddy ecosystems. Thus, repeated pesticide applications are required to control rice planthoppers. Altering the short-term effect formulation of pesticides to a long-acting formulation may be an alternative solution. A pymetrozine controlled-release granule (CRG; $1 \%$ ) was developed by loading the pesticide on bentonite and coating the solid pesticide with resin. Analysis of pymetrozine release indicated that the $1 \%$ pymetrozine CRG release was more than $80 \%$ for 60 days. In the field trial screening, the $1 \%$ pymetrozine CRG showed a controlled effect of $61.96-78.87 \%$ at 48 days after CGR application. Application of $1 \%$ pymetrozine CRG at the recommended dosage and 1.5 times the recommended dosage resulted in terminal residues on brown rice below the maximum residue limit $\left(0.1 \mathrm{mg} \mathrm{kg}^{-1}\right)$ of China and Japan. Moreover, the pesticide granules showed low toxicity against all tested beneficial organisms in the environment. Pymetrozine CRG (1\%) showed good controlled release and efficacy for controlling paddy planthoppers. The compound exhibited a low terminal residue and low toxicity against all tested beneficial organisms. Pymetrozine CRG (1\%) showed great potential for field applications to control paddy planthoppers, because it overcame the rapid loss of biological function during treatment.

Received 24th April 2018 Accepted 3rd June 2018

DOI: $10.1039 / c 8 r a 03516 d$

rsc.li/rsc-advances

\section{Introduction}

Laodelphax striatellus Fallen (small brown planthopper, SBPH), Nilaparvata lugens Stål (brown planthopper, BPH), and Sogatella furcifera Horvath (whitebacked planthopper, WBPH) are major pests in rice-growing areas in Asia. ${ }^{1}$ Continuous outbreaks of rice planthoppers in such regions in China in 2006 (damage of 9.4 million ha), 2007 (damage of 9.4 million ha), and 2011 indicated the importance of redesigning several planthopper management programs. ${ }^{2}$ Moreover, SBPH caused serious rice yield losses by transmitting rice stripe virus disease ${ }^{3}$ and rice black-streaked dwarf virus disease. ${ }^{4}$ A novel viral disease caused by the southern rice black-streaked dwarf virus has recently spread in rice throughout East and Southeast Asia since the

State Key Laboratory Breeding Base of Green Pesticide and Agricultural Bioengineering, Key Laboratory of Green Pesticide and Agricultural Bioengineering, Ministry of Education, Center for Research and Development of Fine Chemicals, Guizhou University, Guiyang 550025, P. R. China. E-mail: xuweiming2009@163. com; Fax: +868518362 0521; Tel: +868518829 2090

$\dagger$ Present work unit: Shangqiu polytechnic, Shangqiu 476000, Henan, P. R. China. mid-2000s. This pathogen has become one of the most important rice pathogens in these regions and is efficiently transmitted by the WBPH in a persistent circulative propagative manner..$^{5-7}$ Chemical control remains the main strategy for planthopper control in subtropical and temperate regions, such as in China, Japan, and South Korea. Although field experiments have demonstrated that unreasonable use of insecticides could also cause planthopper resurgence in subtropical and temperate areas, insecticides are still extensively used in these regions..$^{8-11}$

The most common formulations of chemical, dustable powder, emulsifiable concentrates, suspension concentrate, soluble concentrate, and wettable powders (WP), are applied by dusting or spraying using simple equipment. ${ }^{12}$ These formulations have several disadvantages, such as the rapid release. Moreover, high dosage should be applied, leading to the initial very high concentration of pesticide in the soil, but the efficacy decreases rapidly to a low ineffective level for pest control. ${ }^{13}$ Consequently, these pesticides are applied at much higher doses than needed to overcome losses of the active compound [denoted as active ingredient (a.i)] at the uptake site by 
dissipation and degradation mechanisms and extend the effectiveness of the pesticide for a longer period. Multiple pesticide applications are required to control rice planthoppers. Moreover, runoff and leaching down the soil of the dusted and sprayed formulations has become serious environmental problems and primary sources of surface and groundwater pollution. The total environment of a treated area is exposed to the toxicant, although the pest organism inhabits only a small fraction. ${ }^{14}$ Moreover, common formulations have short residual activity time. Thus, the agent is applied at very higher doses, causing harmful environmental problem. Consequently, longacting and environment-friendly control programs should be developed to deal with planthopper. ${ }^{15}$

Controlled-release (CR) technology is very important in many fields. CR pesticide formulations can be used to gradually deliver the active substance over time for efficient control of pests. These formulations are combinations of pesticide active agent with inert materials that protect and release the active agent over the required time $\mathrm{e}^{\mathbf{1 6 - 1 8}}$ or coating the pesticide active agent with capsules or other organic materials. CR formulation have numerous benefits, including protection of active ingredients from environmental degradation, manipulation of bioavailability and persistence, reduction of toxicity and operator hazards, reduction of phytotoxicity to seeds and crops, reduced agent application rates, and less labor requirement. ${ }^{19-21}$

The present study was performed to propose a pesticide formulation, CR granule (CRG), to control paddy planthopper. The CRG can preserve pesticide stability for long efficacy and guarantee the initial release of the effective dose. The potential of this approach was investigated by encapsulating the model insecticide 1,2,4-triazin-3(2H)-one-4,5-dihydro-6-methyl-4-[(3pyridinylmethylene)amino] (pymetrozine). ${ }^{22}$ This compound is a novel insecticide with selective activity against homopteran insects unrelated to neonicotinoids with a unique mode of action. ${ }^{23}$ The effects of various processing parameters, such as curing time and pesticide content, were investigated. The release rule of the CRG was determined, and field experiment was conducted to study the insecticidal efficacy, the terminal residues and acute toxicity on several beneficial organisms were evaluated too.

\section{Experimental}

\subsection{General}

Unless indicated otherwise, all common reagents and solvents were used as obtained from commercial supplies without further purifications. Epoxy resin (E-44) was procured from Shenzhen Golden Longsheng Technology Co. Ltd. (China). Polyamide resin (605) was procured from Yuanda Chemicals Co. Ltd. (China). Pymetrozine was obtained from Jiangsu Kwin Group Co., Ltd. The core particle of bentonite was obtained from Henan province (China), and bentonite was obtained from Guizhou province (China). The particle strength was determined on a KQ-3 instrument (Yunnan Chemical Research Institute). The pan granulator with heating function was manufactured by the Changzhou Huaxia Drying \& Granulation Equipment Co., Ltd. (China). The compounds were analyzed by HPLC using the
Agilent 1100 series apparatus composed of a quarternary pump, an autosampler, a diode array detector, a vacuum degasser, a column oven, and Agilent Chemstation software. The columns employed reversed-phase column Kromasil ODS-1 C18 (250 mm $\times 4.6 \mathrm{~mm}$ i. d., $5 \mu \mathrm{m}$; Daicel Chemical Industries Ltd.). The injection volume of the analytical samples was $20 \mu \mathrm{L}$. The mobile phases were composed of acetonitrile/water $(20 / 80, v / v)$. Flow rate was set to $1.0 \mathrm{~mL} \mathrm{~min}^{-1}$, and the detection wavelength was fixed at $298 \mathrm{~nm}$, the temperature was kept at $25{ }^{\circ} \mathrm{C}$. The terminal residues of pymetrozine was separated on a Waters ACQUITY ultra-performance liquid chromatography system fitted with a sample manager, a quaternary solvent manager, a PDA detector, and a BEH C18 column $(50.0 \mathrm{~mm} \times 2.1 \mathrm{~mm}$ i. d., 1.7 $\mu \mathrm{m}$ film thickness) from Waters corporation (Massachusetts, USA). $1 \mu \mathrm{L}$ sample solution was injected and the pymetrozine was detected by measurement of absorbance at $298 \mathrm{~nm}$ on a PDA detector. A mixture of methanol, acetonitrile and purified water were used as the mobile phase for gradient elution. The following gradient elution was employed: $10 \%$ methanol and $90 \%$ purified water at the start $1 \mathrm{~min}$, then $10 \%$ acetonitrile and $90 \%$ purified water fort $1.1 \mathrm{~min}$, then $15 \%$ acetonitrile and $85 \%$ purified water for $3.1 \mathrm{~min}$, then $10 \%$ acetonitrile and $90 \%$ purified water fort $1.1 \mathrm{~min}$, then $10 \%$ methanol and $90 \%$ purified water for $1.3 \mathrm{~min}$.

\subsection{Preparation of pymetrozine CRG}

The coated CRG was produced by a pan granulator, and the pan was rotated constantly at approximately $30 \mathrm{rpm}$ all the time, as follows:

(1) Pymetrozine (132.6 g, 95\%), bentonite (3738.6 g), 1dodecanesulfonic acid sodium salt (10 g), and calcium lignosulfonate $(10 \mathrm{~g})$ were mixed and kneaded well.

(2) Epoxy resin $(\mathrm{E}-44,118.8 \mathrm{~g})$ and polyamide resin (605, $118.8 \mathrm{~g}$ ) were diluted by ethanol to $50 \%$ content.

(3) Core particles (7990 g) were charged into the pan granulator. The pan was rotated constantly at approximately $30 \mathrm{rpm}$. The mixed powder in step (1) was added to the core particle controlled by pan granulation, the particles with pymetrozine were used in subsequent processing.

(4) Epoxy resin (E-44, $118.8 \mathrm{~g}, 50 \%)$ and polyamide resin (605, $118.8 \mathrm{~g}, 50 \%$ ) were mixed and homogeneously sprayed over the pymetrozine solid pesticide particles by a spraying nozzle. After resin curing at approximately $95{ }^{\circ} \mathrm{C}$ for $25 \mathrm{~min}, 4080 \mathrm{~g}$ of the particles were taken off from the pan and obtaining the first part of pymetrozine CRG with $1 \%$ resin content.

(5) Then, the rest $7920 \mathrm{~g}$ pymetrozine CRG were continuously sprayed on epoxy resin (E-44, $79.2 \mathrm{~g}, 50 \%)$ and polyamide resin $(605,79.2 \mathrm{~g}, 50 \%)$. After resin curing at approximately $95{ }^{\circ} \mathrm{C}$ for $25 \mathrm{~min}, 4080 \mathrm{~g}$ of the particles were taken off from the pan and obtaining the second part of pymetrozine CRG with $2 \%$ resin content.

(6) Then, the rest $3919 \mathrm{~g}$ pymetrozine CRG was continuously sprayed on epoxy resin (E-44, $39.6 \mathrm{~g}, 50 \%)$ and polyamide resin $(605,39.6 \mathrm{~g}, 50 \%)$. After resin curing at approximately $95{ }^{\circ} \mathrm{C}$ for 25 min, obtaining the third part of pymetrozine CRG with $3 \%$ resin content (about $3960 \mathrm{~g}$ ). 
(7) Uniform mixing the 3 parts of CRG (the first part of $4080 \mathrm{~g}$ CRG taken from step 4, the second part of $4080 \mathrm{~g}$ CRG taken from step 5, the first part of $3960 \mathrm{~g}$ CRG taken from step 6) to finally obtain $1 \%$ pymetrozine CRG. Thus, this process is inexpensive and convenient for application formulation.

\subsection{Pymetrozine content in granule}

Uncoated and resin-coated granule samples $(10 \mathrm{mg})$ were ground to fine powder and quantitatively transferred to a $25 \mathrm{~mL}$ volumetric flask. The volume was made up to $25 \mathrm{~mL}$ with methanol, and the contents were stirred in an ultrasonic bath for $10 \mathrm{~min}$ to completely disintegrate/dissolve the soluble material. After $2 \mathrm{~h}$ at room temperature, the methanolic sample was filtered quantitatively through a $0.45 \mu \mathrm{m}$ millipore filter, and $5 \mu \mathrm{L}$ was injected into the chromatograph column. Analyses were performed in triplicate, and calibration standards were analyzed on the same day as the samples.

\subsection{Analysis of pymetrozine release from CRG}

We adopted two methods to analyze the release rule of $1 \%$ pymetrozine CRG.

Method one is the dissolution test by section water. Pymetrozine CRG $(1 \%, 30 \mathrm{~g})$ and pure water $(1000 \mathrm{~mL}, \mathrm{pH}$ 7.0-7.2) were added into a $1000 \mathrm{~mL}$ jar at $25{ }^{\circ} \mathrm{C}$. The sample water $(10 \mathrm{~mL})$ of the solution was taken from the middle of the jar, the rest of the water $(990 \mathrm{~mL})$ was abandoned, and pure water $(1000 \mathrm{~mL})$ was added. The sample water $(10 \mathrm{~mL})$ ware extracted by dichloromethane $(15 \times 3 \mathrm{~mL})$ and dried by anhydrous sodium sulfate, filtered, and removed the solvent. The residue was dried at $25{ }^{\circ} \mathrm{C}$ and reconstituted by methanol $(2 \mathrm{~mL})$ for HPLC analysis. All experiments were performed in triplicate, the release analyze of $1 \%$ pymetrozine CRG were take on day $1,3,5$, $7,14,21,28,42$, and 60 after sampling.

Method two tests the remaining particle. Pymetrozine CRG $(1 \%, 30 \mathrm{~g})$ was sealed into a millipore nylon net $(150 \mu \mathrm{m})$ and put into a wide-mouthed jar. Then, $1000 \mathrm{~mL}$ of pure water $(\mathrm{pH}$ 7.0-7.2) was added to the jar under constant temperature of $25{ }^{\circ} \mathrm{C}$. The samples in the nylon net were taken out on days 1,3 , $5,7,14,21,28,42$, and 60 and naturally dried at $25^{\circ} \mathrm{C}$. Then, the samples were ground to fine powder and quantitatively transferred to a $25 \mathrm{~mL}$ volumetric flask. The volume was made up to $25 \mathrm{~mL}$ with methanol. The contents were stirred in an ultrasonic bath for $5 \mathrm{~min}$ to completely disintegrate/dissolve the soluble material. After $2 \mathrm{~h}$ at room temperature, the methanolic sample was then filtered quantitatively through a millipore filter $(0.45 \mu \mathrm{m})$. Then, $5 \mu \mathrm{L}$ of the sample was injected into the chromatograph column. Analyses were performed in triplicate.

\subsection{Insecticide field trials of $1 \%$ pymetrozine CRG}

Field tests were conducted at Libo country, Guizhou province, China, in July and August of 2012. Twenty-four field plots $\left(20 \mathrm{~m}^{2}\right)$ with medium fertility were planted to test the insecticide activity. The plots were isolated from each other to avoid cross-contamination. Prior to the application of $1 \%$ pymetrozine CRG, the rice field had a water layer, which was $4-5 \mathrm{~cm}$ deep. The water layer was kept for 2-4 days after pesticide application. Four insecticide treatments and a blank water control were performed as follows: (1) $1 \%$ pymetrozine CRG at $75 \mathrm{~g}$ ai per ha, broadcasted uniformly to the field surface with $0.25 \mathrm{~kg}$ of urea fertilizer; (2) $1 \%$ pymetrozine CRG at $150 \mathrm{~g}$ ai per ha, broadcasted uniformly to the field surface with $0.25 \mathrm{~kg}$ of urea fertilizer; (3) $1 \%$ pymetrozine CRG at $300 \mathrm{~g}$ ai per ha, broadcasted uniformly to the field surface with $0.25 \mathrm{~kg}$ of urea fertilizer; (4) $1 \%$ pymetrozine CRG at $450 \mathrm{~g}$ ai per ha, broadcasted uniformly to the field surface with $0.25 \mathrm{~kg}$ of urea fertilizer; (5) control group, 25\% pymetrozine WP at $93.75 \mathrm{~g}$ ai per ha, sprayed by a manual sprayer (Shandong Wish Plant Protection Machinery Co., LTD, China) operated at a pressure of $2 \mathrm{~kg} \mathrm{~cm}^{-2}$; (6) only water as blank control. Randomized block design with four replications was used. Diagonal sampling was adopted at five positions, and 25 rice clusters were investigated for each position. The planthoppers were collected with a basin while the rice clusters were flapped gently. Insecticide efficacy was calculated as follows: [(sum of planthoppers in the blank control area - sum of planthopper in the pesticide application area)/sum of planthopper in the blank control area] $\times 100$.

\subsection{Terminal residues of pymetrozine in the soil, rice straw, rice husk, and brown rice}

Based on previously described methods (NY/T788-2004, Guideline on pesticide residue trials) ${ }^{24}$ the field trials were conducted in three experimental fields in Guizhou (red soil, $\mathrm{pH}$ 5.5), Guangxi (yellow brown soil, pH 6.8), and Heilongjiang (black mud, pH 6.7) during the agricultural season in 2012 and 2013. Each field was divided into $30 \mathrm{~m}^{2}$ blocks for the control and treatment groups in the dissipation rate study. To investigate the distribution of terminal residue of pymetrozine in the soil, rice straw, rice husk, and brown rice, $1 \%$ pymetrozine CRG was applied at doses of $450 \mathrm{~g}$ ai per ha and $675 \mathrm{~g}$ ai per ha, which were the recommended dosage and 1.5 times the recommended dosage, respectively. Soil samples were collected from soil layer depths of $0-15 \mathrm{~cm}$ at harvest time. Rice straw, rice husk, and brown rice samples were collected into polyethylene bags at harvest time, transported to the laboratory, and stored at $-20{ }^{\circ} \mathrm{C}$ until analysis.

Portions of the homogenized soil (10 g), rice straw (10 g), and rice husk $(5 \mathrm{~g})$ samples were weighed into a $150 \mathrm{~mL}$ conical flask and extracted with $60 \mathrm{~mL}$ of acetone/water $(\mathrm{v} / \mathrm{v}=8 / 2$, by volume, and containing $1 \%$ ammonia). The mixture was vibrated for $60 \mathrm{~min}$ on a reciprocating shaker. The mixture was filtered through Celite ${ }^{\circledR} 545$ and washed with $20 \mathrm{~mL}$ of acetone/water $(\mathrm{v} / \mathrm{v}=8 / 2$, by volume, and containing $1 \%$ ammonia). The extract was collected and pooled. Acetone was removed under reduced pressure. Potassium carbonate ( $3 \mathrm{~g}$ ) and sodium chloride ( $3 \mathrm{~g}$ ) were added, and the mixture was extracted with dichloromethane $(2 \times 50 \mathrm{~mL})$. The dichloromethane layer was dried with anhydrous $\mathrm{Na}_{2} \mathrm{SO}_{4}$, and organic solvent was removed under reduced pressure. The residue was dissolved in $1 \mathrm{~mL}$ of methanol, after purification by PSA $(0.05$ g) and anhydrous magnesium sulfate $(0.1 \mathrm{~g})$. The solution was filtered with a $0.22 \mu \mathrm{m}$ nylon filter (Millipore, Billerica, MA, USA) and subjected to UPLC. 
A portion of the chopped brown rice $(10 \mathrm{~g})$ was weighed into a $50 \mathrm{~mL}$ polytetrafluoroethylene tube and extracted with acetonitrile $(20 \mathrm{~mL})$ and ammonium hydroxide $(6 \mathrm{~mL}$, $0.1 \mathrm{~mol} \mathrm{~L}^{-1}$ ). After vortex oscillation for $4 \mathrm{~min}$, sodium chloride $(4.0 \mathrm{~g})$ was added to the solution. The sample was again vortex oscillated for $2 \mathrm{~min}$ and centrifuged for $5 \mathrm{~min}$ at $6000 \mathrm{rpm}$ to obtain $10 \mathrm{~mL}$ of the supernatant. The solvent was removed under reduced pressure. For future determination, the residue was dissolved in $1 \mathrm{~mL}$ of methanol. After purification by PSA $(0.05 \mathrm{~g})$ and anhydrous magnesium sulfate $(0.1 \mathrm{~g})$, the solution was filtered with a $0.22 \mu \mathrm{m}$ nylon filter (Millipore, Billerica, MA, USA) and subjected to UPLC.

\subsection{Acute toxicity of $1 \%$ pymetrozine CRG on some beneficial organisms in the environment}

Acute toxicity was determined based on previously described methods (GB/T31270, Test guidelines on environmental safety assessment for chemical pesticides) ${ }^{25}$ recommended by the Standardization Administration of the People's Republic of China and General Administration of Quality Supervision, Inspection and Quarantine of the People's Republic of China and proposed by the Ministry of Agriculture of the People's Republic of China. Acute toxicity tests were performed according to GB/ T31270 (Part 9, avian acute toxicity test; Part 10, honeybee acute toxicity test; Part 12, fish acute toxicity test; Part 13, Daphnia sp. acute immobilization test; Part 14, alga growth inhibition test; Part 15, earthworm acute toxicity test) on Apis mellifera L., Coturnix coturnix japonica, Brachydanio rerio, Daphnia magna Straus, Selenastrum capricornutum, and Eisenia foetida. Toxicity grades of pesticides on silkworm were defined as extremely toxic, highly toxic, moderately toxic, or lowly toxic according to the $\mathrm{LC}_{50}, \mathrm{EC}_{50}$, or $\mathrm{LD}_{50}$ values (GB/T31270-9, 10, 12, 13, 14, and 15).

\subsection{Statistical analysis}

Pymetrozine content and release were analyzed using Excel. Data from the field tests were statistically analyzed by ANOVA using SPSS (PASW Statistic 18). Duncan's multiple tests were applied to calculate the significant differences among the CR of the blends at $5 \%$ level $(P=0.05)$.

\section{Results and discussion}

\subsection{Preparation of $1 \%$ pymetrozine CRG}

Pymetrozine is a fat-soluble pesticide, in the water, surfactant 1dodecanesulfonic acid sodium salt and calcium lignosulfonate ensure that pymetrozine reaches the crop. The characteristics of the $1 \%$ pymetrozine CRG are presented in Table 1 . The core granules were generally spherical in shape. The white powder technical-grade pymetrozine was readily mixed with the bentonite matrix and then added to the core particle. This process resulted in larger and heavier granules with more spherical, higher weight and less aggregation.

When the epoxy resin (E-44) and polyamide resin (605) were mixed in one system, single molecules (monomers) of the resins combined to form long chains of molecules (polymers). As the mixture was cured, the sample became a hard polymer. The
Table 1 The effect of curing time and temperature on some physical properties of $\mathrm{CRG}^{a}$

\begin{tabular}{lllrl}
\hline Entry & $\mathrm{T} /{ }^{\circ} \mathrm{C}$ & Time/min & Hardness/N & Curing quality \\
\hline 1 & 70 & 90 & $7.62 \pm 0.32$ & Sticky \\
2 & 80 & 60 & $7.9 \pm 0.57$ & A little sticky \\
3 & 90 & 30 & $8.39 \pm 0.45$ & Solidifying \\
4 & 100 & 25 & $9.02 \pm 0.46$ & Solidifying
\end{tabular}

${ }^{a} 30 \mathrm{CRG}$ particles (with $2 \%$ resin content) were randomly selected for hardness test.

hardened, finished polymers were almost nontoxic, and the insecticide ingredient pymetrozine was sealed. We optimized the curing conditions, and Table 1 shows that, out of the four entries, entry 4 afforded the best result in terms of quality.

\subsection{Pymetrozine content in granule}

First, we tested the accuracy and precision of the method adopted. Pymetrozine standard was dissolved in absolute methanol, and pymetrozine samples $(0.099,0.198$, $1.98 \mu \mathrm{g} \mathrm{mL}^{-1}$ ) were prepared. The accuracy and precision of the determination were tested, $y=71.128 x-919.91, \gamma=0.9996$. The average recovery rate was $90.32 \%, 90.37 \%$ and $90.97 \%$, the RSD were $1.27,0.92$ and 2.30, Therefore, this method is suitable to test the pymetrozine content of CRG.

A controlled-drug-release carrier should have the capacity to encapsulate a large amount of drug to prolong the release and reduce the quantity of carrier required for application. The hollow structure of bentonite facilitates its entrapment of more pymetrozine. To determine the amount of pymetrozine entrapped and resin encapsulated, as well as test the uniform distribution, we investigated the pymetrozine content of uncoated granule sample and cured, resin-coated granule sample. The results showed that the initial pymetrozine content was $1.10 \%$, which decreased to $1.03 \%$ after coating and curing. The results indicated that bentonite could entrap pymetrozine, and the resins did not adsorb pymetrozine. Therefore, resin can be used to develop CR formulation of pymetrozine.

\subsection{Analysis of pymetrozine release from $1 \%$ pymetrozine CRG}

The Pesticide Fact Sheet of pymetrozine by the United States Environmental Protection Agency (EPA) showed that the hydrolysis time (half-life) of pymetrozine is $<14$ days ( $\mathrm{pH} \mathrm{5,} 25$ ${ }^{\circ} \mathrm{C}$ ), $>80$ days (pH 7), and $>86$ days (pH 9). Solubility of this compound in water is $0.29 \mathrm{~g} \mathrm{~L}^{-1}\left(\mathrm{pH}\right.$ 6.4-6.5, $25{ }^{\circ} \mathrm{C}$ ). Thus, pymetrozine hydrolyses readily at low $\mathrm{pH}$, and the water qualities have strong influence on the stability of pymetrozine.

Li et al. studied the residue behavior of pymetrozine in the paddy field in the main rice production region of China (Hunan and Zhejiang province), the results showed that the dissipation rates of pymetrozine in rice water were fast with half-life of 7-9 days. ${ }^{24}$ Yang developed a systematic study on pymetrozine residues in rice and environmental media by combining laboratory and field trials. The results showed that the half-life of pymetrozine was less than two days in Henan and Hunan 
province..$^{25}$ The degradation of pymetrozine was generally fast, and this pesticide belongs to the easily degraded pesticide in Chinese main rice production region. The degradation of $1 \%$ pymetrozine CRG cannot be ignored because of its long-acting formulation. To avoid the influence of degradation, we tested the release condition of $1 \%$ pymetrozine CRG in two methods. Method 1 was designed to measure the amount of cumulative release amount from the granule, by testing the content of pymetrozine in water. Method 2 was designed to measure the amount of pymetrozine remaining in the granule and calculate the release amount of pymetrozine (Fig. 1).

On days $1,3,5,7,14,21,28,42$, and 60 after preparation of the test system, we tested the released amount of pymetrozine. For method 1 , the released amount at certain days were as follows: day $1,11.04 \%$; day $5,26.89 \%$, day $14,44.21 \%$, which was almost half of the total amount; and day $28,60.87 \%$; The release rate of pymetrozine decreased with prolonged time. The cumulative released amounts on days 42 and 60 were $72.33 \%$ and $80.32 \%$, respectively. For method 2, on day 1 , the remaining and released amounts were $87.6 \%$ and $12.4 \%$, respectively. On day 5 , the remaining particles were $74.84 \%$. On day 14 , the remaining particles were $54.45 \%$, while the corresponding release was $45.55 \%$, which was almost half of the total amount. Meanwhile, the remaining particles on day 28 were $37.22 \%$. The release rate of pymetrozine decreased with prolonged time. On days 42 and 60 , the remaining particles were $25.1 \%$ and $14.23 \%$, respectively, and the corresponding released amounts were $74.9 \%$ and $85.77 \%$, respectively.

The release test demonstrated that the release period of the CRG formulation lasted for more than 60 days. The release period could be divided into three stages with the fastest release on days $1-2$, during which the release amount was more than $15 \%$. The medium-release stage was from 3 days to 28 days, with cumulative release amount of approximately $50 \%$. The remaining

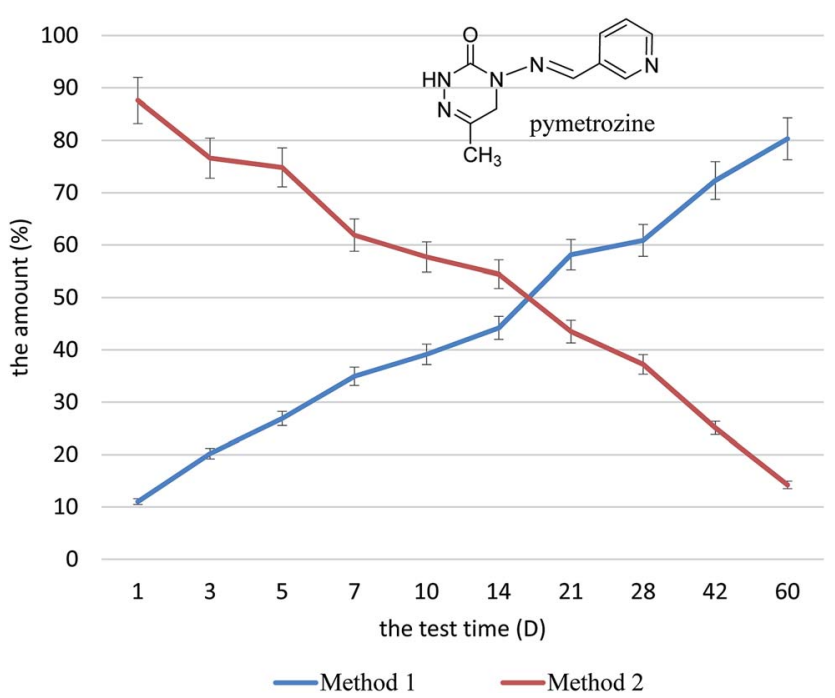

Fig. 1 The cumulative release of pymetrozine (\%) from resin-based CRG at different time. Method 1 was designed to measure the amount of cumulative release amount (\%) from the granule by testing the content of pymetrozine in water at different time (day). Method 2 was designed to measure the amount (\%) of pymetrozine remaining in the granule at different time (day). pymetrozine was released during the slow stage. These results demonstrate that the $\mathrm{CR}$ formulation could release the active ingredient from the composition at desired timing and desired properties with extraordinarily good CR property of active ingredients. Application of pymetrozine CRG in the paddy during the "before-heading period" could effectively control planthopper by quickly increasing the pymetrozine concentration in the paddy water during the fastest release period. The long-term releasing properties of pymetrozine could control the subsequent periods.

\subsection{Insecticide field trials of $\mathbf{1 \%}$ pymetrozine CRG}

In the field trial screening, the insecticidal efficacy of $1 \%$ pymetrozine CRG against $S$. furcifera in Guizhou was evaluated. The CR formulations were broadcasted to the field surface after 9 days of rice transplanting. The results are provided in Table 2 in terms of control effect values.

At doses of 75, 150, 300, and $450 \mathrm{~g}$ ai per ha, the control effects changed with time, with the control effects on certain days after CRG application as follows: day 14, 65.83-84.40\%; day 21, 72.71$96.8 \%$; day $28,72.71-86.62 \%$ control effects; days $38,75.68-$ $87.02 \%$; and day $48,61.96-78.87 \%$. At $450 \mathrm{~g}$ ai per ha, the effect ranged from $78.87 \%$ to $88.21 \%$ at $14-48$ days after CRG application. By contrast, dose of $300 \mathrm{~g}$ ai per ha resulted in $77.44 \%$ to $86.38 \%$ effect for the same period. The persistence period of $1 \%$ pymetrozine CRG was apparently approximately 48 days. For $25 \%$ pymetrozine WP at $375 \mathrm{~g}$ ai per ha, the persistence period was approximately 21 days, and the effect ranged from $12.48 \%$ to $46.17 \%$ at $28-48$ days after spraying. Moreover, the effects of $300 \mathrm{~g}$ ai per ha and $450 \mathrm{~g}$ ai per ha showed no significant difference on day 48. However, the effects of these doses showed significant difference with those of $75 \mathrm{~g}$ ai per ha and $150 \mathrm{~g}$ ai per ha at all investigated times of 48 days after CRG application.

\subsection{Terminal residues of pymetrozine in the soil, rice straw, rice husk, and brown rice}

Terminal residue levels of pymetrozine in the soil, rice straw, rice husk, and brown rice are listed in Table 3.

We have mix pymetrozine standard with the matrix material, such as soil, rice straw, rice husk, brown rice, the RSD $3.25 \pm$

Table 2 The field insecticidal efficiency of 1\% pymetrozine CRG against $S$. furcifera

\begin{tabular}{|c|c|c|c|c|c|c|c|c|c|c|}
\hline \multirow[b]{2}{*}{ Entry } & \multicolumn{2}{|l|}{14 days } & \multicolumn{2}{|c|}{21 days } & \multicolumn{2}{|c|}{28 days } & \multicolumn{2}{|c|}{38 days } & \multicolumn{2}{|c|}{48 days } \\
\hline & Effect $^{a}$ & $\mathrm{Sig}^{b}$ & Effect & Sig & Effect & Sig & Effect & Sig & Effect & Sig \\
\hline 1 & 65.83 & $\mathrm{Ab}$ & 72.71 & $\mathrm{Bb}$ & 5.68 & $\mathrm{Aa}$ & 76.07 & $\mathrm{Bb}$ & 1.96 & $\mathrm{Aa}$ \\
\hline 2 & 69.73 & $\mathrm{Ab}$ & 82.39 & $\mathrm{Ab}$ & 82.72 & $\mathrm{Aa}$ & 83.62 & $\mathrm{Aa}$ & 73.93 & Aa \\
\hline 3 & 79.41 & Aab & 83.63 & $\mathrm{Aa}$ & 84.14 & $\mathrm{Aa}$ & 86.38 & $\mathrm{Aa}$ & 77.44 & $\mathrm{Aa}$ \\
\hline 4 & 84.40 & Aab & 86.62 & $\mathrm{Aa}$ & 87.02 & $\mathrm{Aa}$ & 88.21 & $\mathrm{Aa}$ & 78.87 & Aa \\
\hline 5 & 90.80 & Aa & 86.27 & $\mathrm{Aa}$ & 46.17 & $\mathrm{Bb}$ & 23.09 & $\mathrm{Cc}$ & 12.48 & $\mathrm{Bb}$ \\
\hline
\end{tabular}

${ }^{a}$ Efficiency (\%). ${ }^{b} \mathrm{Sig}=$ significance of difference, the statistical analysis was conducted by DMRT method at the condition of equal variances assumed $(p=0.05)$. Entry 1, 2, 3, and 4 mean $75 \mathrm{~g}$ ai per ha, $150 \mathrm{~g}$ ai per ha, $300 \mathrm{~g}$ ai per ha, and $450 \mathrm{~g}$ ai per ha of pymetrozine in CRG formulation, entry 5 means $25 \%$ pymetrozine WP at $93.75 \mathrm{~g}$ ai per ha. 
Table 3 Terminal residues of pymetrozine in soil, rice straw, rice husk, and brown rice in Guizhou, Guangxi and Heilongjiang in 2012 and 2013 $\left(\mathrm{mg} \mathrm{kg}^{-1}\right)^{a}$

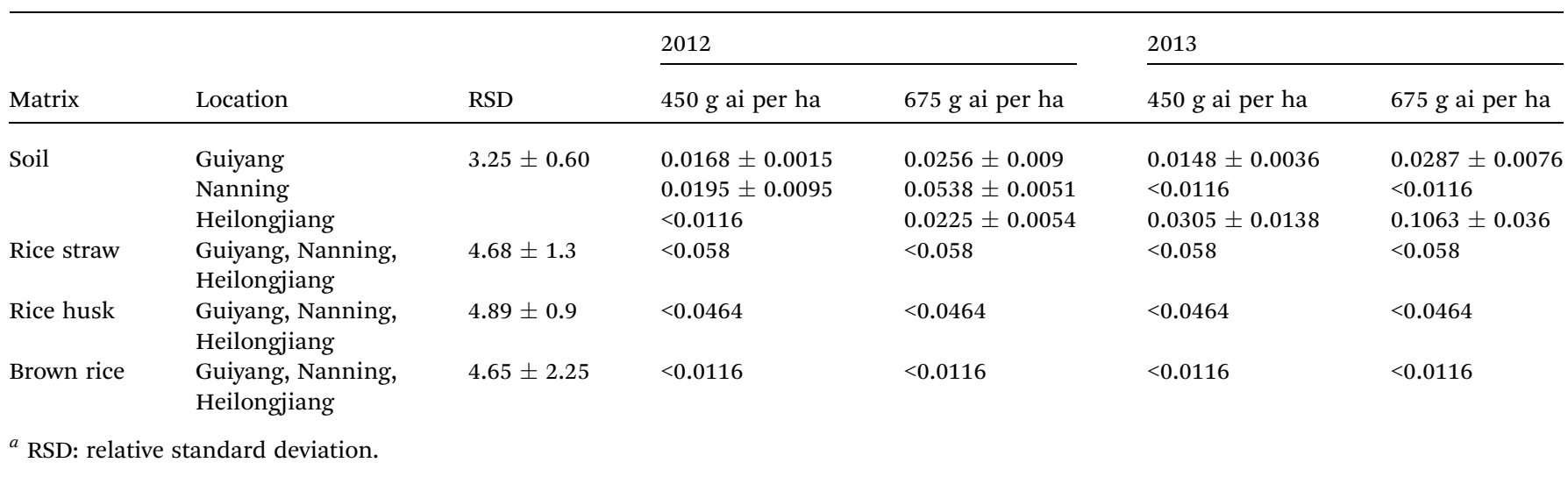

Table 4 Acute toxicity of 1\% pymetrozine CRG on some beneficial organisms in environment in vitro

\begin{tabular}{|c|c|c|}
\hline Living organism & Test guidelines & The result at exposure time \\
\hline Coturnix coturnix japonica & 9 , acute oral toxicity & $>66.8 \mathrm{mg}$ ai per $\mathrm{kg}$ bw, $168(\mathrm{~h})\left(\mathrm{LD}_{50}\right)$ \\
\hline Apis mellifera $\mathrm{L}$. & 10, acute inhaling toxicity & $>11.0 \mu \mathrm{g}$ ai per bee, $48(\mathrm{~h})\left(\mathrm{LD}_{50}\right)$ \\
\hline Brachydanio rerio & 12 , acute oral toxicity & $>100 \mathrm{mg}$ ai per $\mathrm{L}, 96(\mathrm{~h})\left(\mathrm{LD}_{50}\right)$ \\
\hline Daphnia magna Straus & 13 , acute immobilisation test & $>100 \mathrm{mg}$ ai per L, $48(\mathrm{~h})\left(\mathrm{EC}_{50}\right)$ \\
\hline Selenastrum capricornutum & 14 , growth inhibition test & >100 mg ai per L,72 (h) $\left(\mathrm{EC}_{50}\right)$ \\
\hline
\end{tabular}

$0.60,4.68 \pm 1.3,4.89 \pm 0.9,4.65 \pm 2.25$, respectively. At one time application of $450 \mathrm{~g}$ ai per ha (the recommended dosage), the terminal residue levels of pymetrozine in the soil at harvest ranged from $<0.0116 \mathrm{mg} \mathrm{kg}^{-1}$ to $<0.058 \mathrm{mg} \mathrm{kg}^{-1}$. When applied at $675 \mathrm{~g}$ ai per ha (1.5 times the recommended dosage), the terminal residue levels ranged from $<0.0116 \mathrm{mg} \mathrm{kg}^{-1}$ to $0.1063 \mathrm{mg} \mathrm{kg}^{-1}$ in the soil. Terminal residue levels of pymetrozine in the rice straw, rice husk, and brown rice were $<0.058$, $<0.0464$, and $<0.0116 \mathrm{mg} \mathrm{kg}^{-1}$, respectively, when pymetrozine was applied at 450 and $675 \mathrm{~g}$ ai per ha. In China and Japan, the MRL on brown rice was $0.1 \mathrm{mg} \mathrm{kg}{ }^{-1}$. Hence, at the recommended dosage and 1.5 times the recommended dosage, the terminal residue were below the MRL. This result indicated the safety of $1 \%$ pymetrozine CRG application on rice at the recommended dosage.

\subsection{Acute toxicity of $1 \%$ pymetrozine CRG on several beneficial organisms in environment}

Under standard procedures, we tested the acute inhaling toxicity, acute oral toxicity, acute immobilization toxicity, and growth inhibition toxicity against the beneficial organisms in the environment. These organisms included A. mellifera L., $C$. coturnix japonica, B. rerio, D. magna Straus, S. capricornutum, and $E$. foetida, and the results are listed in Table 4.

The acute inhaling toxicity against $A$. mellifera $\mathrm{L}$ indicated $\mathrm{LD}_{50}$ of $>11.0 \mu \mathrm{g}$ ai per bee. The acute oral toxicity against $A$. mellifera L. C. coturnix japonica, B. rerio, and E. foetida showed $\mathrm{LD}_{50}$ values of $>2000 \mathrm{mg}$ ai per $\mathrm{L},>66.8 \mathrm{mg}$ ai per $\mathrm{kg}$ bw,
$>100 \mathrm{mg}$ ai per $\mathrm{L}$ and >100 mg ai per kg dry ground, respectively. The acute immobilization test against $D$. magna Straus showed $\mathrm{EC}_{50}$ of $>100 \mathrm{mg}$ ai per L. The growth inhibition test against Selenastrum capricornutum showed $\mathrm{EC}_{50}$ of $>100 \mathrm{mg}$ ai per L. In the "Fact Sheet for Pymetrozine" published by the United States Environmental Protection Agency, demonstrate that pymetrozine has been determined to be of low acute toxicity to humans, birds, aquatic organisms, mammals. For example, the acute inhaling toxicity against northern bobwhite quail (Colinus virginianus) indicated $\mathrm{LD}_{50}$ of $>2000.0 \mathrm{mg} \mathrm{kg}{ }^{-1}$, and categorized to practically nontoxic. Furthermore, the pymetrozine content in CRG is only $1 \%$. These results indicated that $1 \%$ pymetrozine CRG had low toxicity against all tested beneficial organisms in the environment.

\section{Conclusions}

The effectiveness time of most chemical pesticides are not long enough to control rice planthoppers. Altering traditional agrichemical formulations into formulations with longer efficacy may be an alternative solution. We prepared 1\% pymetrozine CRG using bentonite and resin. Analysis of the pymetrozine release indicated that the $\mathrm{CR}$ formulation of pymetrozine had good release property. In the field trial screening, 1\% pymetrozine CRG showed good efficacy for controlling paddy planthopper, with control effect of $61.96-78.87 \%$ at 48 days. At the recommended dosage and the 1.5 times of the recommended dosage, the terminal residues in brown rice remained below the MRL (0.1 $\mathrm{mg} \mathrm{kg}^{-1}$ ) in China and Japan. Moreover, 1\% 
pymetrozine CRG showed low toxicity against all tested beneficial organisms in the environment. Thus, 1\% pymetrozine CRG demonstrated great potential for field applications to control paddy planthopper, because it overcame the rapid loss of biological function during treatment.

\section{Conflicts of interest}

There are no conflicts to declare.

\section{Acknowledgements}

The authors gratefully acknowledge the financial support of the National Key Research and Development Plan (No. 2016YFD0200707), National Natural Science Foundation of China (No. 31760532), Special Fund for Agro-Scientific Research in the Public Interest (No. 201203022). Guizhou S\&T Project (No. 201462, 20113071, 2013137), for the financial support. The authors thank Mr Changyu Shao (plant protection and quarantine station, Guizhou province) who carried out the field trials experiments in this study. The authors thank National Center for the Quality Supervision \& Test of Pesticides (Beijing) for acute toxicity on several beneficial organisms.

\section{References}

$1 \mathrm{~J}$. A. Cheng, in Planthoppers: new threats to the sustainability of intensive rice production systems in Asia, ed. K. Heong and B. Hardy, International Rice Research Institute, Los Baños (Philippines), 2009, pp. 157-178.

2 J. A. Cheng, J. L. Zhu, Z. R. Zhu and L. G. Zhang, J. Environ. Entomol., 2008, 2, 016.

3 L. J. Cai, X. Z. Ma, L. Kang, K. J. Deng, S. Y. Zhao and C. B. Li, J. Virol. Methods, 2003, 112, 115-120.

4 X. C. He, H. X. Xu, X. S. Zheng, Y. J. Yang, G. C. Gao, J. H. Pan and Z. X. Lu, Rice Sci., 2012, 19, 335-338.

5 Z. Chen, C. J. Yin, J. J. Liu, M. J. Zeng, Z. C. Wang, D. D. Yu, L. Bi, L. H. Jin, S. Yang and B. A. Song, Arch. Virol., 2012, 157, 2327-2333.

6 D. D. Yu, Z. C. Wang, J. Liu, M. M. Lv, J. J. Liu, X. Y. Li, Z. Chen, L. H. Jin, D. Y. Hu, S. Yang and B. A. Song, J. Agric. Food Chem., 2013, 61, 8049-8055.
7 G. H. Zhou, D. L. Xu, D. G. Xu and M. X. Zhang, Front Microbiol., 2013, 4, 270.

8 T. Nagata, T. Kamimuro, Y. C. Wang, S. G. Han and N. M. Noor, J. Asia-Pac. Entomol., 2002, 5, 113-116.

9 S. Y. Li, X. Liu, C. F. Gao, X. P. Bo, J. Y. Su, Y. H. Wang, L. Yu, X. Yan, J. L. Shen and J. Yang, Chin. J. Rice Sci., 2009, 23, 7984.

10 H. B. Bao, S. H. Liu, J. H. Gu, X. Z. Wang, X. L. Liang and Z. W. Liu, Pest Manage. Sci., 2009, 65, 170-174.

11 S. F. Jin, M. G. Feng, S. H. Ying, W. J. Mu and J. Q. Chen, Pest Manage. Sci., 2011, 67, 36-43.

12 F. Quaglia, F. Barbato, G. De Rosa, E. Granata, A. Miro and M. I. La Rotonda, J. Agric. Food Chem., 2001, 49, 4808-4812.

13 J. I. Perez-Martinez, E. Morillo, C. Maqueda and J. M. Gines, Pest Manage. Sci., 2001, 57, 688-694.

14 L. X. Wen, Z. Z. Li, H. K. Zou, A. Q. Liu and J. F. Chen, Pest Manage. Sci., 2005, 61, 583-590.

15 T. Sanchez-Verdejo, T. Undabeytia, S. Nir, C. Maqueda and E. Morillo, Environ. Sci. Technol., 2008, 42, 5779-5784.

16 M. D. Lopez, A. Maudhuit, M. J. Pascual-Villalobos and D. Poncelet, J. Agric. Food Chem., 2012, 60, 1187-1192.

17 Z. J. Zhou, Y. Z. Shen, C. W. Du, J. M. Zhou, Y. S. Qin and Y. J. Wu, Land Degrad. Dev., 2017, 28, 2370-2379.

18 A. Rashidzadeh, A. Olad and M. J. Hejazi, Adv. Polym. Technol., 2017, 36, 177-185.

19 R. Gil-Ortiz, Pest Manage. Sci., 2015, 71, 1685-1693.

20 J. X. Liu, Q. Zhao and X. G. Zhang, Appl. Clay Sci., 2017, 145, 44-52.

21 A. Watanabe, Y. Takebayashi, T. Ohtsubo and M. Furukawa, Pest Manage. Sci., 2009, 65, 1233-1240.

22 B. Sechser, B. Reber and F. Bourgeois, J. Pestic. Sci., 2002, 75, 72-77.

23 H. Mercan, E. Yilmaz and R. Inam, J. Hazard. Mater., 2007, 141, 700-706.

24 Ministry of Agriculture and Rural Affairs, PRC, standard, 2004, NY/T 788, 13.

25 Ministry of Agriculture and Rural Affairs, PRC, standard, 2014, GB/T31270/9-15, 68. 